\title{
Molecular Motions of Poly( $\gamma$-monosubstituted-benzyl D-glutamate) in the Solid State
}

\author{
Naoki SASAKI, ${ }^{* 1}$ Akihiro TsuTSUMI, ${ }^{* 1}$ Kunio HIKICHI, ${ }^{* 1, * 3}$ \\ Yasuo KonISHI, ${ }^{* 2}$ and Masahiro HATANO*2 \\ ${ }^{* 1}$ Department of Polymer Science, Faculty of Science, \\ Hokkaido University, Sapporo 060, Japan. \\ ${ }^{* 2}$ Chemical Research Institute of Nonaqueous Solutions, \\ Tohoku University, Katahira, Sendai 980, Japan.
}

(Received February 23, 1979)

\begin{abstract}
Dielectric measurements were performed at frequencies ranging from $30 \mathrm{~Hz}$ to $300 \mathrm{KHz}$ and at temperatures between -150 and $120^{\circ} \mathrm{C}$ for the following poly $(\gamma$-monosubstitutedbenzyl D-glutamate)s; poly( $\gamma$-o-chlorobenzyl D-glutamate) ( $o$-ClPBDG), poly $(\gamma-m$-chlorobenzyl Dglutamate) ( $m$-ClPBMDG), poly $(\gamma$-p-chlorobenzyl D-glutamate) ( $p$-ClPBDG), poly $(\gamma-p$-nitrobenzyl D-glutamate) ( $p$-NPBDG), poly ( $\gamma$ - $p$-methylbenzyl D-glutamate) ( $p$ MePBDG) and a racemic mixture of $p$-CIPBDG and $p$-CIPBLG ( $p$-ClPBDG $+p$-ClBLG). Each sample exhibited a dielectric loss maximum in the temperature range of $30-90^{\circ} \mathrm{C}$. These loss maxima were attributed to the onset of large scale motion of side chains. The temperatures of maximum loss (the dispersion temperature) are shifted by the introduction of substituents. The dispersion temperature is highest for $p$-CIPBDG among three monochlorosubstituted PBDGs. For $p$-monosubstituted-PBDGs, $p$-NPBDG, which has the side chain with the largest dipole moment among three $p$-substituents studied, showed the highest dispersion temperature. The results are explained in terms of restriction caused by dipole-dipole interaction among dipoles in the side chain. The enormous relaxation strength of $o$ CIPBDG was considered to reflect a certain conformation of the side chain originating from steric hindrance between the ester and the $o$-substituent of a side chain. Racemic mixtures of $p$-CIPBDG and $p$-ClPBLG showed no evidence of the first-order solid-solid transition observed for racemic mixtures of PBDG and PBLG.

KEY WORDS Poly( $\gamma$-monosubstituted-benzyl D-glutamate) / Side-Chain Motion / Dipole-Dipole Interaction / Steric Hindrance / Racemic Mixtures /
\end{abstract}

It has widely been accepted that even in the solid state, long side chains of the $\alpha$-helical poly $(\alpha$-amino acid) undergo considerable motions while the $\alpha$ helical main chain remains rigid. The importance of side-chain motions of the $\alpha$-helical poly $(\alpha$-amino acid) in determining properties in the solid state has been recognized. ${ }^{1-9}$

Recently, it has been reported that in a series of poly $(\gamma$-monosubstituted-benzyl D-glutamate)s, in which a substituent is introduced into the phenyl ring at the end of the side chain of poly $\gamma$-benzyl Dglutamate) (PBDG), the properties markedly depend on the species and the position of the substituent. ${ }^{10-12}$ It has been also reported that the different position of the substitution affects the

*3 To whom all inquiries should be addressed. conformation of the $\alpha$-helical backbone. ${ }^{13,14}$ Though qualitatively, the dependence of the helical structure and its stability on the species of the side chain has been explained in terms of side chain-side chain and side chain-backbone interactions. ${ }^{8}$

The purpose of the present investigation is to examine the effects of the substitution to the benzyl group of PBDG on side-chain motions of PBDG. Dielectric and broad-line nuclear magnetic resonance measurements were performed to observed molecular motions for the following seven samples of $\operatorname{poly}(\gamma$-monosubstituted-benzyl glutamate)s; poly $(\gamma$--chlorobenzyl D-glutamate) (o-ClPBDG), poly $(\gamma-m$-chlorobenzyl D-glutamate) ( $m$-ClPBDG), poly $(\gamma-p$-chlorobenzyl D-glutamate) ( $p$-ClPBDG), poly( $\gamma$-p-chlorobenzyl L-glutamate) ( $p$-CIPBLG), poly $(\gamma-p$-nitrobenzyl D-glutamate) ( $p$-NPBDG), 
poly $(\gamma-p$-methylbenzyl D-glutamate) ( $p$-MePBDG), and the racemic mixture of $p$-ClPBDG and $p$ ClPBLG ( $p$-CIPBDG $+p$-CIPBLG). In particular, $p$ $\mathrm{ClPBDG}+p-\mathrm{ClPBLG}$ was investigated from the point of view of phenyl ring stacking since such a stacking has been reported to occur for a racemic mixture of PBLG and PBDG. ${ }^{15-20}$

The results are compared with those of PBLG and discussed in relation to the effects of the substitution.

\section{EXPERIMENTAL}

All samples except for two polymers of $p$-CIPBDG and $p$-CIPBLG were prepared by the ester exchanging reaction of poly( $\gamma$-methyl D-glutamate) with corresponding alcohols. ${ }^{12,21}$ The degree of substitution was checked by proton NMR spectra and elemental analysis for carbon. The degree of substitution was above $90 \%$ for $o$-CIPBDG, $m$ CIPBDG, and $p$-NPBDG, and $66 \%$ for $p$-MePBDG. $p$-CIPBDG and $p$-CIPBLG were synthesized by the method of Ledger, et al. ${ }^{22,23} p-\mathrm{ClPBDG}+p$ CIPBLG was prepared by dissolving equi-moles of $p$ CIPBDG and $p$-CIPBLG in chloroform. Films for dielectric measurements of $o$-CIPBDG, $m$-CIPBDG, $p$-CIPBDG, and $p$-MePBDG were prepared by casting concentrated chloroform solutions on a glass plate at room temperature. A film of $p$-NPBDG was made from concentrated $m$-cresol solution at $50^{\circ} \mathrm{C}$. Silver electrodes were vacuum-deposited on both surfaces of film to assure complete electric contact. Each film was set in the measuring cell and evacuated at a temperature of $150^{\circ} \mathrm{C}$ until the dielectric constant remained unchanged.

The dielectric constant and loss factor were measured on an Ando Denki TR-1C bridge with a lock-in amplifier of NF Circuit Design Block Co., Ltd. and an Ando Denki WBG-5 oscillator. Measurements were made over a frequency ranging from $30 \mathrm{~Hz}$ to $300 \mathrm{kHz}$ and within temperatures ranging from -150 to $120^{\circ} \mathrm{C}$.

Samples used for broad-line NMR measurements were freeze-dried powders from dioxane solutions. Before making measurements, each sample was evacuated at a temperature of $100^{\circ} \mathrm{C}$ for $48 \mathrm{~h}$ to eliminate residual solvent.

A JEOL JNS-B spectrometer was employed for obtaining derivatives of broad-line proton NMR absorption curves. The temperature was varied from that of liquid nitrogen to about $100^{\circ} \mathrm{C}$.

\section{RESULTS}

$o-C l P B D G, m-C l P B D G$, and $p-C l P B D G$

Figure 1 shows the temperature dependence of the dielectric constant $\varepsilon^{\prime}$ and the loss factor $\varepsilon^{\prime \prime}$ measured at a frequency of $1 \mathrm{kHz}$ for $o$-CIPBDG, $m$-CIPBDG, and $p$-CIPBDG. The data for PBLG are also shown for comparison. For each sample, a remarkable dielectric dispersion was observed above room temperature. The dispersion observed for PBLG has been interpreted in terms of the onset of side-chain metions. ${ }^{3}$ NMR,,${ }^{4,7,24}$ dynamic mechanical, ${ }^{24,25}$ and dielectric $^{6,24}$ measurements for PBLG and other polyglutamates have provided much evidence for supporting this interpretation. Thus, it is reasonable to consider that dielectric dispersion observed above room temperature for the samples of monochloroPBDG are attributed to the same origin as PBLG.

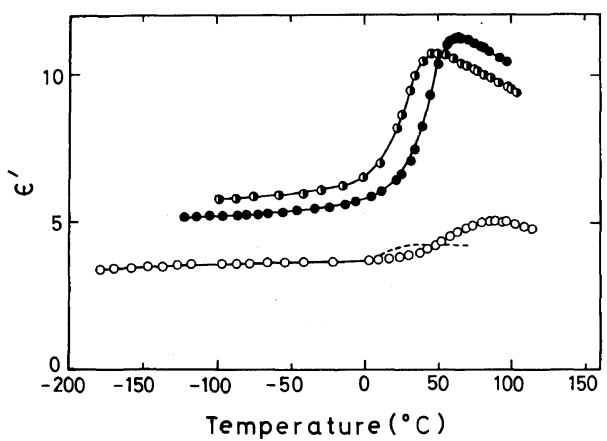

(a)

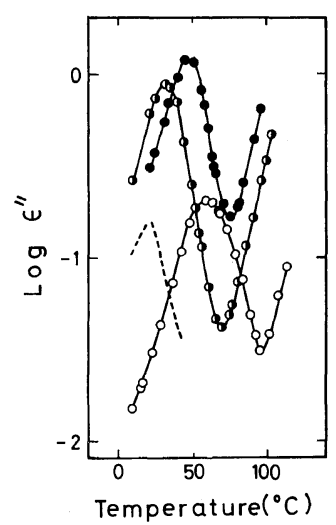

(b)

Figure 1. Temperature dependence of (a) the dielectric constant and (b) the loss factor of $o$-CIPBDG $(\bullet), \mathrm{m}^{-}$ CIPBDG (๑), p-CIPBDG (○), and PBLG (---) measured at $1 \mathrm{kHz}$. 
The dielectric constant was of the order $m$ $\mathrm{ClPBDG}>o-\mathrm{ClPBDG}>p-\mathrm{ClPBDG} \simeq \mathrm{PBLG}$ in the temperature range of $-50-0^{\circ} \mathrm{C}$.

Figure 2 shows the results of NMR measurements for $o$-ClPBDG, $m$-ClPBDG, and $p$-ClPBDG; the second moment of the absorption line is plotted against temperature. Experimental points were omitted for the sake of clarity. Data for PBLG are also shown in the figure by a dotted line. It was found that the second moments for all samples studied decrease with increasing temperature in two steps. In the temperature range from -140 to $-20^{\circ} \mathrm{C}$, the second moments decrease gradually with increasing temperature. The decrease in second moment in this temperature range may be attributed to the rotation of the phenyl ring as is the case of PBLG. ${ }^{4.7,24}$ Above room temperature, all samples show an abrupt decrease in the second moment which is related to the appearence of the dielectric side-chain dispersion in the same temperature range.

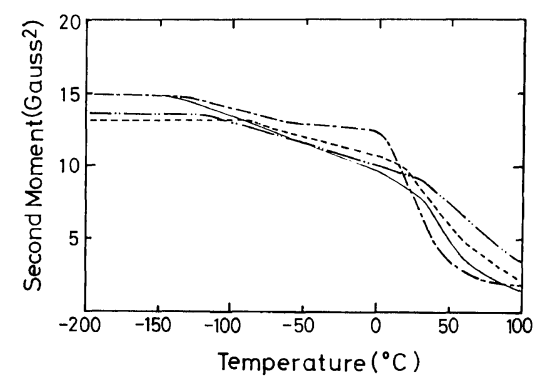

Figure 2. The second moment of monochloro-PBDGs as a function of temperature: ------, $o$-CIPBDG; - - -,$m$ ClPBDG; - - - $p$-CIPBDG; - PBLG.

Figure 3 shows the frequency dependence of $\varepsilon^{\prime}$ and $\varepsilon^{\prime \prime}$ for $o$-CIPBDG at various temperatures from 21.5 to $70.2^{\circ} \mathrm{C}$. It is apparent that $o$-CIPBDG shows a dielectric dispersion in this temperature range. All other samples show similar frequency dependence as $o$-ClPBDG.

In Figure 4, the temperature dependence of the relaxation strength $\Delta \varepsilon=\varepsilon_{0}-\varepsilon_{\infty}$ is shown for $o$ ClPBDG, $m$-ClPBDG, $p$-CIPBDG, and PBLG. The relaxation strength was determined by the Cole-Cole plot. It was found that the relaxation strength is of the order $o$-ClPBDG $>m$-ClPBDG $>p$-ClPBDG $\simeq$ PBLG, and that a maximum appears in the $\Delta \varepsilon$-temperature curve.

After normalizing $\left(\varepsilon^{\prime}-\varepsilon_{\infty}\right)$ - and $\varepsilon^{\prime \prime}$-frequency curves by $\Delta \varepsilon$, all data could be superposed on a

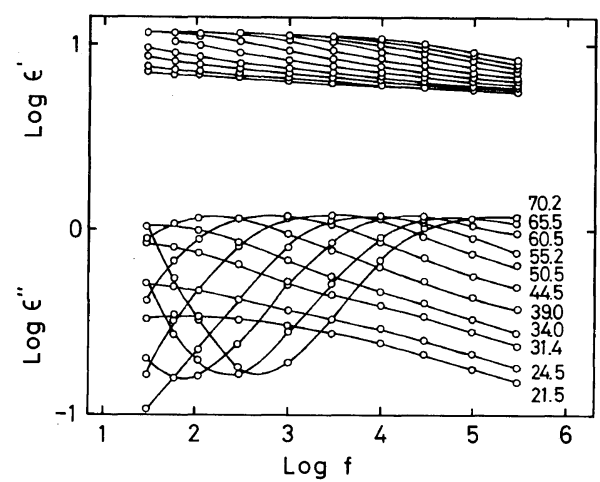

Figure 3. The dielectric constant and the loss factor for $o$-CIPBDG as a function of logarithm of frequency at indicated temperatures.

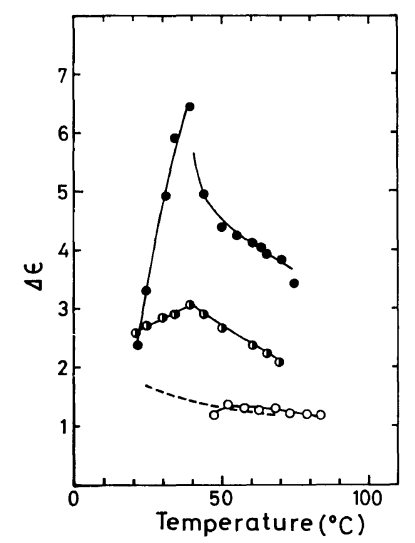

Figure 4. Temperature dependence of the relaxation strength obtained from the Cole-Cole plot of monochloro-PBDGs: $o$-ClPBDG (@), $m$-ClPBDG (๑), $p$ CIPBDG (O), and PBLG (---).

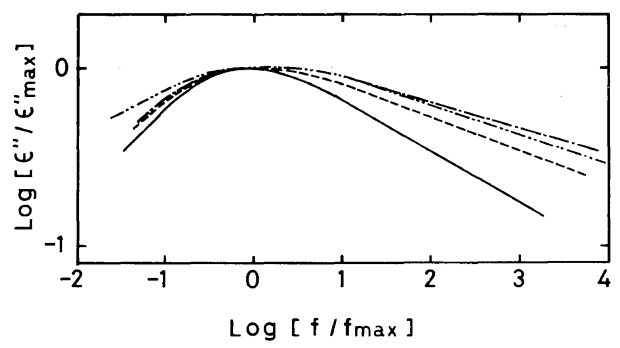

Figure 5. Composite master curves of the loss factor for monochloro-PBDGs: --.--, o-ClPBDG; -- $\quad$, $m$ CIPBDG; --- - $p$-CIPBDG; ——, PBLG.

master curve at a certain temperature by performing suitable horizontal translations along the logarithmic frequency axis. The master curves of the loss 
factor for $o$-CIPBDG, $m$-CIPBDG, $p$-ClPBDG, and PBLG are compared in Figure 5, where the normalized loss factor is represented in units of its maximum value and the reduced frequency in units of the value at which the maximum occurs. It is apparent that the substitution of chlorine atoms at the phenyl ring of PBDG causes the dispersion to

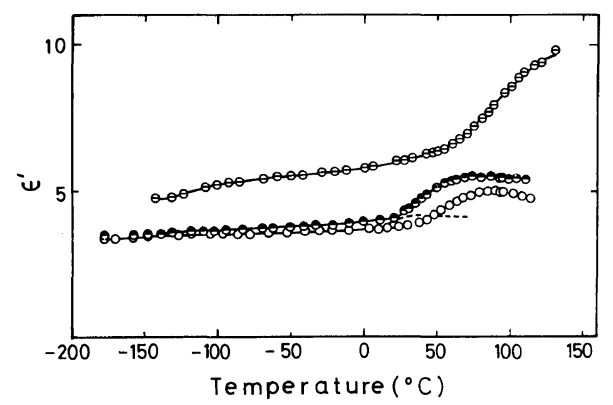

(a)

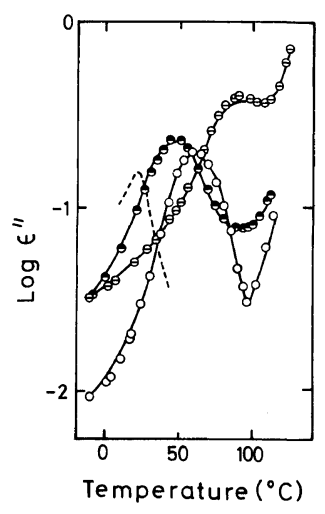

(b)

Figure 6. Temperature dependence of (a) the dielectric constant and (b) the loss factor of $p$-NPBDG $(\Theta), p$ MePBDG ( $\Theta$ ), $p$-CIPBDG $(\bigcirc)$, and PBLG (---), measured at $1 \mathrm{kHz}$.

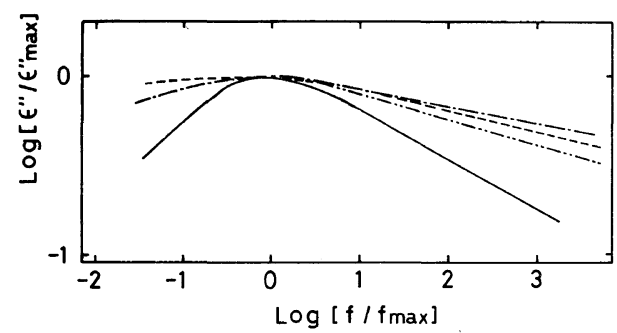

Figure 7. Composite master curves of the loss factor for p-monosubstituted PBDGs: ------, p-NPBDG; - - -, pMePBDG; - - - $p$-ClPBDG; — $\longrightarrow$ PBLG. become much broader.

$p-N P B D G, p-C l P B D G$, and $p-M e P B D G$

Figure 6 shows the temperature dependence of the dielectric constant $\varepsilon^{\prime}$ and the loss factor $\varepsilon^{\prime \prime}$ measured at $1 \mathrm{kHz}$ for $p$-NPBDG, $p$-CIPBDG, $p$-MePBDG, and PBLG. Each sample clearly shows a dielectric dispersion attributable to side chain motions for the same reason as described previously for monochloro-PBDG. Considerably higher dispersion temperature of $p$-NPBDG implies that there exist strong constraints on side-chain motions.

Master curves of the loss factor for $p$-NPBDG, $p$ ClPBDG, $p$-MePBDG, and PBLG, obtained by the same procedure as described in the preceding section, are compared in Figure 7. It is apparent that the master curve of the loss factor is much broader for $p$ substtituted PBDGs than for PBLG. Among monosubstituted PBDGs studied here, $p$-NPBDG and $p$ MePBDG show much broader dispersion curves.

$p-C l P B D G$ and $p-C l P B D G+p-C l P B L G$

Figure 8 shows the temperature dependence of the

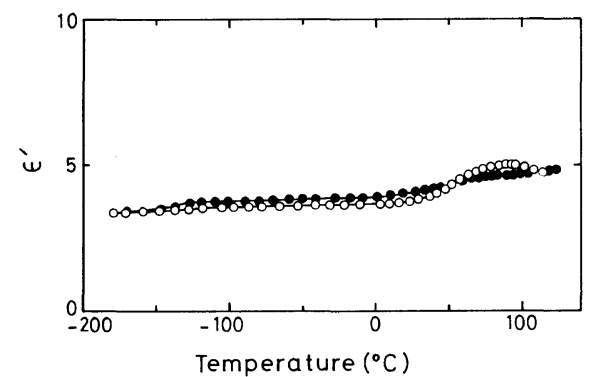

(a)

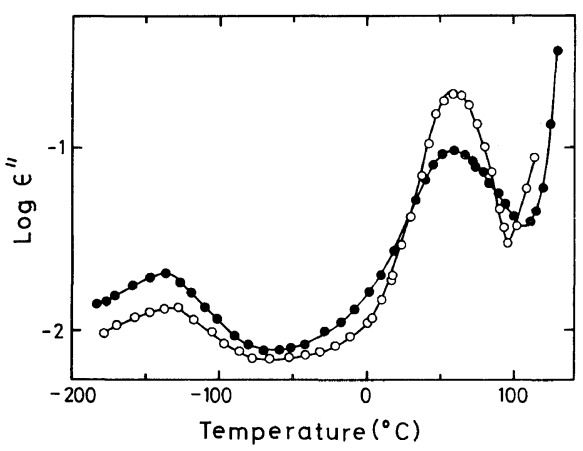

(b)

Figure 8. Temperature dependence of (a) the dielectric constant and (b) the loss factor of $p$-CIPBDG $(O)$ and $p$ $\mathrm{ClPBDG}+p-\mathrm{CIPBLG}(\bigcirc)$ measured at $1 \mathrm{kHz}$. 
dielectric constant $\varepsilon^{\prime}$ and the loss factor $\varepsilon^{\prime \prime}$ measured at $1 \mathrm{kHz}$ for $p$-CIPBDG and $p$-ClPBDG $+p$ CIPBLG. In each sample, two dielectric dispersions are observed, one located at about $60^{\circ} \mathrm{C}$ (dispersion I) and the other located far below room temperature (dispersion II). As already mentioned dispersion I is assigned to the onset of the large scale motion of side chain, while dispersion II is considered due to the vibrational local motion of the side chain. ${ }^{24,28,29}$

As for the dispersion temperature of dispersion I, the two samples show the same temperature of $60^{\circ} \mathrm{C}$, on the other hand, for dispersion II, $p$-CIPBDG $+p$ CIPBLG shows a dispersion temperature lower than $p$-ClPBLG by some $10^{\circ} \mathrm{C}$. Such lowering of the dispersion temperature of dispersion II by racemic mixing has been also reported for PBDG + PBLG. ${ }^{30}$

In Figure 9 is shown the temperature dependence of the relaxation strength $\Delta \varepsilon$ obtained by the Cole-Cole plot. $\Delta \varepsilon$ for the racemic mixture increases monotonously with increasing temperature, while for $p$-ClPBDG, $\Delta \varepsilon$ vs. temperature curve has a maximum at a certain temperature. The value of $\Delta \varepsilon$ of the racemic mixture is smaller than that of $p$ CIPBDG.

The time-temperature superposition principle was also applicable for dispersion I of $p$-ClPBDG $+p$ CIPBLG. The results of $p$-CIPBDG and $p$ -

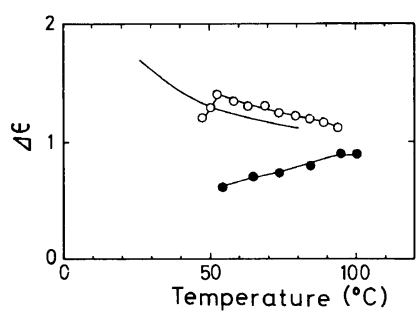

Figure 9. Temperature dependence of the relaxation strength obtained from the Cole-Cole plot of $p$-CIPBDG (○) and $p$-ClPBDG $+p$-ClPBLG (O).

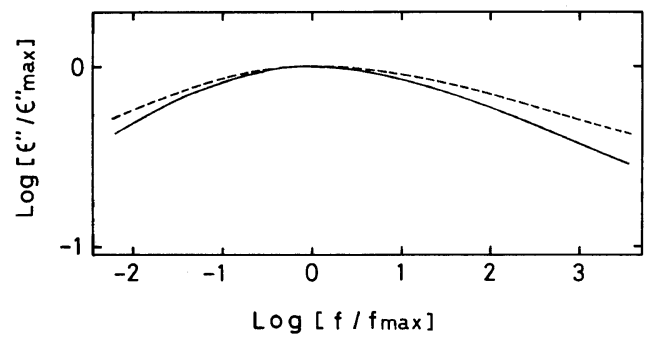

Figure 10. Composite master curves of the loss factor of $p$-CIPBDG (-) and $p$-CIPBDG $+p$-CIPBLG (-----).
$\mathrm{ClPBDG}+p-\mathrm{ClPBLG}$ are shown in Figure 10, of which the ordinate and abscissa are the same as in Figure 5. The master curve for $p-\mathrm{ClPBDG}+p$ ClPBLG is found to be broader than that for $p$ ClPBDG.

\section{DISCUSSION}

The appearance of a maximum in the $\Delta \varepsilon$-temperature curve for all samples except for $p$ ClPBDG $+p$-CIPBLG suggests that the observed dispersion for each sample has a property reminiscent of the glass transition of the synthetic amorphous polymer. This suggestion was also intimated by the temperature dependence of the shift factor $b_{\mathrm{T}}$ obtained by the superposition procedure. It was found that $b_{\mathrm{T}}$ follows the WLF equation..$^{25,26}$

Table I shows the dispersion temperature $T_{\max }$ which is defined as the temperature of the loss maximum at a frequency of $1 \mathrm{kHz}$ for monosubstituted PBDG along with PBLG for comparison. The dispersion temperatures of all monosubstituted PBDG are higher than that of PBLG. This implies that the introduction of the substituent into the phenyl ring gives rise to restrictions on side-chain motions. The broadened master curves of monosubstituted PBDG in comparison with that of PBLG also suggest the existence of such a restriction.

The influence of the introduction of the substituent into the phenyl ring on molecular motions has been reported for polystyrene. ${ }^{31,32}$ Barb pointed out various ways by which the softening point of polystyrene can be elevated. (I) Substitution for specifically causing intramolecular steric hindrance

Table I. Dielectric dispersion temperature

\begin{tabular}{ll}
\hline \multirow{1}{*}{ Sample } & $T_{\max }{ }^{\mathrm{a}}$ \\
\cline { 2 - 2 } & ${ }^{\circ} \mathrm{C}$ \\
\hline$o-\mathrm{ClPBDG}$ & 47 \\
$m$-ClPBDG & 32 \\
$p$-ClPBDG & 60 \\
$p$-NPBDG & 90 \\
$p-\mathrm{MePBDG}$ & 47 \\
$p$-ClPBDG $+p$-CIPBLG & 60 \\
PBDG & 25
\end{tabular}

a $T_{\max }$, dielectric dispersion temperature defined as the temperature of the loss maximum at a frequency of $1 \mathrm{kHz}$. 
between the substituent and vicinal main chain segments; large rigid $o$-substituents produce such an effect. (II) Substitution to increase steric hindrance between the phenyl ring and the neighboring polymers. Large rigid groups in an "exposed" position, particularly the $p$-position, have this effect. (III) Substitution by dipolar groups to produce longrange dipole-dipole interaction, as in the case of poly( $p$-chlorostyrene).

This explanation can be used to advantage. In our case, $T_{\max }$ may be taken as a measure of the restriction acting on side-chain motions. It is in the following order: for monochloro PBDGs, $p$ ClPBDG $\left(60^{\circ} \mathrm{C}\right)>o$-ClPBDG $\left(47^{\circ} \mathrm{C}\right)>m$ CIPBDG $\left(32^{\circ} \mathrm{C}\right)>$ PBLG $\left(25^{\circ} \mathrm{C}\right)$, and for $p$-monosubstituted PBDGs, $p$-NPBDG $\left(90^{\circ} \mathrm{C}\right)>p$ CIPBDG $\left(60^{\circ} \mathrm{C}\right)>p$-MePBDG $\left(47^{\circ} \mathrm{C}\right)$.

The highest $T_{\max }$ for $p$-ClPBDG in the former series indicates that (II) is a dominant effect, while for polystyrene, (I) is most effective. The difference in structure between polystyrene and the side chain of PBDG may be responsible for the lesser effectiveness of (I) in the present case. In the former, the phenyl ring has biforked polymer segments at its root, but in the latter, there is only one side-chain segment.

The estimation of allowed conformations of the side chain of monochloro-PBDGs was made by the Monte Carlo calculation using an electronic computer. ${ }^{33}$ The results showed that for $p$-CIPBDG, the chlorine atom is likely to be located in outermost parts of the side-chain region surrounding the $\alpha$ helical backbone, while for $o$-CIPBDG, the chlorine atom is located more or less in the inner parts of the side-chain region. Thus, the dipole-dipole interaction between "exposed" dipoles of neighboring molecules for $p$-CIPBDG may be more effective than the steric hindrance between the substituent and the other part of the side chain.

The enormous relaxation strength of $o$-CIPBDG suggests that the side chain of $o$-ClPBDG may take a certain conformation with a large resultant dipole moment by the short range correlation of the $\mathrm{C}=\mathrm{O}$ dipole and the $\mathrm{C}-\mathrm{Cl}$ dipole. Scheraga et al. have calculated the minimum energy-conformation of the side chain of poly( $\beta$-o-chlorobenzyl aspartate), which has a shorter side chain than $o$-CIPBDG by one methylene group but otherwise is very similar. ${ }^{34}$ A conformation proposed by these gives a value of 2.7 Debye for the dipole moment of the side chain, which is obviously larger than the value of 1.4 Debye caclulated without considering the correlation of $\mathrm{C}=\mathrm{O}$ and $\mathrm{C}-\mathrm{Cl}$ dipoles.

The highest $T_{\max }$ of $p$-NPBDG indicates the existence of strong contributions from the long range dipole interaction and the sizable volume of the nitro group to the restriction on side-chain motions. The effect may be enhanced by the substitution at the "exposed" position. In Figure 11, the dispersion temperature $T_{\max }$ is plotted against the mean square of the dipole moment of $p$-substituted phenyl rings in order to examine the correlation between the polarity and the restriction on the side-chain motions.

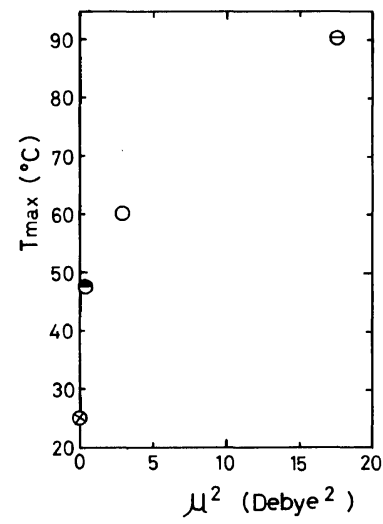

Figure 11. The dispersion temperature $T_{\max }$ plotted against the square of dipole moment of substituted benzyl groups; that of $p$-N PBDG $(\ominus)$, of $p$-ClPBDG ( $\bigcirc)$, of $p$ $\operatorname{MeOBDG}(\Theta)$, and of PBLG $(\otimes)$.

The value of the dipole moment was taken from a literature. ${ }^{27}$ It is found that there is a definite correlation between values of $T_{\max }$ and the mean square of the dipole moment $\left\langle\mu^{2}\right\rangle$. These results suggest that the contribution of dipole-dipole interaction to the restriction affecting side-chain motions are appreciable.

As mentioned in the results section, the liberation of the phenyl ring rotation around the benzyl methylene carbon and phenyl ring carbon bond $\left(C_{\zeta}-C_{\phi}\right)$ occurs at lower temperatures. Since the decrement of the second moment in the temperature range between -140 and $0^{\circ} \mathrm{C}$ is almost the same for each polymer, the phenyl ring in each sample is likely to rotate in a similar way. The effect of the liberation of rotation of the phenyl ring upon the dielectric constant depends on the position of the substitution of polar group. In the case of $p$-CIPBDG, since the direction of the $\mathrm{C}-\mathrm{Cl}$ dipole agrees with the rotation axis, the phenyl ring rotation should be ineffective 
changing the direction of the dipole moment, and the dielectric constant should be equal to that of PBLG in a temperature range where only rotation of $\mathrm{C}_{\zeta}-\mathrm{C}_{\phi}$ occurs. But, in the case of $o$-CIPBDG and $m$ ClPBDG, the contribution from the $\mathrm{C}_{\zeta}-\mathrm{C}_{\phi}$ dipole to the dielectric constant is significant. The somewhat smaller value of $o$-ClPBDG in comparison with that of $m$-isomer seems to be caused by the steric hindrance between the $\mathrm{O}-\mathrm{C}=\mathrm{O}$ and $\mathrm{C}-\mathrm{Cl}$ groups.

Racemic mixture of PBDG and PBLG $(\mathrm{PBDG}+\mathrm{PBLG})$ has been reported to show an extra meridional X-ray reflection of $10.6 \AA$ attributable to the phenyl ring stacks in the side chains. ${ }^{15-20}$ The previous dielectric measurements showed that the first-order phase transition due to the break down of the stacks occurs at about $90^{\circ} \mathrm{C}$ for PBDG + PBLG. For a racemic mixture of $p$-CIPBDG and $p$-CIPBLG, no reflections reminiscent of the stack were observed. ${ }^{13}$ Furthermore, we could not observe any evidence for the first-order transition in this study. The large dipole moment and large volume of chlorine atoms seem to prevent stacking for $p$ $\mathrm{ClPBDG}+p$-CIPBLG. The dispersion temperatures of the dispersion I for $p$-CIPBDG and for $p$ $\mathrm{ClPBDG}+p$-CIPBLG are almost the same. The relaxation strength for $p$-ClPBDG $+p$-CIPBLG, however, is smaller than that of $p$-ClPBDG and monotonously increases with temperature. It is likely that the dipole-dipole interaction between "exposed" dipoles is more effective for $p$-CIPBDG $+p$ ClPBLG than for $p$-ClPBDG.

\section{CONCLUSIONS}

The dielectric dispersion of monosubstituted PBDG indicates that the introduction of substituents to the phenyl ring of the side chain brings about strong interaction between the side chains. This interaction is most remarkable for $p$-substitution in three monochloro-PBDGs and can be explained mainly in terms of the dipole-dipole interactions among the "exposed" $\mathrm{C}-\mathrm{Cl}$ dipoles of $p$-ClPBDG. The restriction upon side-chain motions is enhanced by the increase in polarity and bulkiness of the substituent. For $o$-CIPBDG, a steric hindrance between the $o-\mathrm{Cl}$ and the carboxyl group determines a side-chain conformation which gives the large resultant dipole moment. For a racemic mixture of $p$ CIPBDG and $p$-ClPBLG, no evidence of phenyl ring stacks of the side chain was observed, although such stacks are believed to exist for PBDG + PBLG.

Acknowledgments. This work was partly supported by the Grant-in-Aid for Scientific Research from the Ministry of Education, Culture, and Science of Japan. The authors are grateful to Mr. M. Osanai for supplying the samples of $p$-CIPBDG and $p$-ClPBLG, and to Mr. H. Shimodate for his careful dielectric measurements on racemic mixtures of $p-\mathrm{Cl}$ PBDG and $p$-CIPBLG.

\section{REFERENCES}

1. R. G. Saba, J. A. Sauer, and A. E. Woodward, J. Polym. Sci., A, 1, 1843 (1963).

2. J. A. E. Kail, J. A. Sauer, and A. E. Woodward, $J$. Phys. Chem., 66, 1292 (1963).

3. K. Hikichi, K. Saito, M. Kaneko, and J. Furuichi, $J$. Phys. Soc. Jpn., 19, 557 (196).

4. K. Hikichi, J. Phys. Soc. Jpn., 19, 2169 (1964).

5. S. Makino, K. Kamashima, S. Kubota, and S. Sugai, Jpn. J. Appl. Phys., 3, 55 (1964).

6. A. Tsutsumi, K. Hikichi, M. Mitsumaki, and M. Kaneko, J. Phys. Soc. Jpn., 22, 1120 (1967).

7. A. Tsutsumi, Jpn. J. Appl. Phys., 9, 2225 (1970).

8. M. Chien, E. T. Samulski, and C. G. Wade, Macromolecules, 6, 638 (1973).

9. H. A. Arfmann, R. Labitzke, and K. G. Wagner, Biopolymers, 14, 1381 (1975).

10. Y. Konishi and M. Hatano, J. Polym. Sci. Polym. Lett. Ed., 14, 303 (1976).

11. Y. Konishi and M. Hatano, J. Polym. Sci. Polym. Lett. Ed., 14, 351 (1976).

12. Y. Konishi and M. Hatano, J. Polym. Sci. Polym. Lett. Ed., 14, 2329 (1976).

13. M. Osanai, A. Tsutsumi, K. Hikichi, M. Kaneko, Y. Konishi, and M. Hatano, Rep. Prog. Polym. Phys. Jpn., 19, 517 (1976)

14. M. Osanai, A. Tsutsumi, K. Hikichi, Y. Konishi, and M. Hatano, Rep. Prog. Polym. Phys. Jpn., 20, 589 (1977).

15. M. Tsuboi, A. Wada, and N. Nagashima, J. Mol. Biol., 3, 705 (1961).

16. A. Elliott, R. D. B. Fraser, and T. P. MacRae, J. Mol. Biol., 11, 821 (1965).

17. Y. Mitsui, Y. Iitaka, and M. Tsuboi, J. Mol. Biol., 24, 15 (1967)

18. J. M. Squire and A. Elliott, J. Mol. Biol., 65, 291 (1972).

19. T. Takahashi, A. Tsutsumi, K. Hikichi, and M. Kaneko, Macromolecules, 7, 806 (1974).

20. N. Matsushima, K. Hikichi, A. Tsutsumi, and M. Kaneko, Polym. J., 7, 382 (1975).

21. E. M. Bradbury, B. G. Carpenter, and H. Goldman, Biopolymers, 6, 837 (1968). 
22. R. Ledger and F. H. C. Stewart, Aust. J. Chem., 18, 1477 (1965).

23. R. Ledger and F. H. C. Stewart, Aust. J. Chem., 19, 1729 (1966).

24. A. Tsutsumi, K. Hikichi, T. Takahashi, Y. Yamashita, N. Matsushima, M. Kanke, and M. Kaneko, J. Macromol. Sci.-Phys., B8, 413 (1973).

25. Y. Yamashita, A. Tsutsumi, K. Hikichi, and M. Kaneko, Polym. J., 8, 114 (1975).

26. M. L. Williams, R. F. Landel, and J. D. Ferry, J. Am. Chem. Soc, 77, 3701 (1955).

27. L. Pauling, "Nature of the Chemical Bond," Cornell
University Press, Ithaka, New York, N.Y., 1960.

28. K. Yamafuji, J. Phys. Soc. Jpn., 15, 2295 (1960).

29. R. Hayakawa and Y. Wada, J. Polym. Sci. Polym. Phys. Ed., 12, 2119 (1974).

30. K. Shimizu, O. Yano, and Y. Wada, Rep. Prog. Polym. Phys. Jpn., 18, 547 (1975).

31. W. G. Barb, J. Polym. Sci., 37, 515 (1959).

32. R. D. McCammon and R. G. Saba, J. Polym. Sci., Part A-2, 7, 1721 (1969).

33. K. Hikichi, unpublished results.

34. J. F. Yan, F. A. Momang, and H. A. Scheraga, J. Am. Chem. Soc., 92, 1109 (1970). 